\title{
Misconceptions regarding dental health care: A cross sectional study
}

\author{
Manmeet Kour ${ }^{1, *}$, Nidhi Puri Narang ${ }^{2}$, Akansha Budakoti ${ }^{3}$, Akshay Rthore ${ }^{4}$, Anuridhi Choudhary ${ }^{5}$, Prateek \\ Aggarwal $^{6}$
}

1,3,5,6 PG Student, ${ }^{2}$ Reader, ${ }^{4}$ Senior Lecturer, Dept. of Oral Medicine and Radiology, I. T.S. Centre for Dental Studies \& Research, Muradnagar, Ghaziabad, Uttar Pradesh, India

*Corresponding Author:

Email: manmeetkour000@gmail.com

\begin{abstract}
Introduction: In dentistry there are many misconceptions and other improven beliefs which passed from one generation to another. Many of these misconceptions had a significant impact on oral health of population. These misconceptions usually emerge from fake traditional beliefs and non-scientific knowledge. Lack of education is also a major factor which make people believe in these misconceptions.

Aim: The aim of this cross sectional self-assessed questionnaire was to assess the prevalence of misconceptions about dentistry in the population of district Murad Nagar U.P.

Materials and Method: A self-assessed questionnaire consisting of 20 questions was provided to the patients visiting the OPD in Department of Oral Medicine and Radiology, to assess the prevalence of misconceptions about dentistry. A total of 200 individuals were included in the study and data was collected using a validated four-part questionnaire including demographic data, questions regarding dental problems, oral cancer, dental treatments and miscellaneous misconceptions.

Results: The results were subjected to statistical analysis after the completion of survey. The results revealed that myths and misconceptions associated with dental treatments were significantly higher among females as compared to males. The people in age group above 50 were found to have more misconceptions as compared to other age groups. Similarly less educated and the people with less income were found to have more misconceptions.

Conclusion: Misconceptions regarding dental health still persists in the minds of general population which needs rectification by constant education and awareness regarding quality of dental services provided to enmass.
\end{abstract}

Keywords: Misconception, Dental health care, Myths.

\section{Introduction}

Misconceptions has no relevance with the reality and is commonly held but a false belief, a myth or a fictitious or imaginary understanding of a thing or a person. These are generally deep-rooted invariably form part and parcel of a society for longer periods and are very difficult to be separated apart often. Misconceptions breed on human's ignorance, and unknown superstitiousness, and imagination. ${ }^{1}$

This is true in every aspect of our life, but it is more prominent having far greater intrinsic importance in case of our health-care domain. As such it is indispensably required to fully ward off the misconceptions for the obvious reason of our health as it is the most important and prominent of our all priorities. ${ }^{1}$

The incidence of oral health problems across most age groups in the community requires a range of community and targeted individual responses. ${ }^{2}$ These misconceptions can be prevalent in a population due to a variety of reasons like poor education and cultural beliefs. ${ }^{1}$ Misconceptions related to oral diseases and oral health-related practices are very common in Indian population. These misconceptions are further firmly fixed in the psyche of future generation over a space of time. $^{3}$

Gradually with the development of education, these misconceptions are disappearing, but still they persist and are commonly encountered and the field of dentistry is no exception to these myths. ${ }^{4}$

It is difficult to break this chain as it is deep-seated in the society and understanding them becomes essential to provide a good care. ${ }^{1}$ In the field of dentistry, most beliefs (no proven fact) guide the patients in the wrong protocol which can lead to difficulty for dentist to provide a satisfying treatment and effect management outcome adversely. ${ }^{5}$

There are many dental misconceptions, some are child related, some of them adult related and the rest superstitious. This study was therefore conducted to identify and resolve some of these myths. ${ }^{4}$

Hence, the aim of this cross sectional self-assessed questionnaire was to assess the prevalence of misconceptions about dentistry in the population of district Murad Nagar U.P

\section{Materials and Method}

A cross-sectional study was conducted among the outpatients attending a dental institute in U.P, in order to assess the prevalence of misconceptions regarding dental health.

Source of data: The data was obtained from a survey, which included a questionnaire based on the misconceptions that were related to dentistry and was received by the patients.

Study population: The study population included all the 
patients which attended dental institute and patients willing to participate in the study. Based on age and gender of the patients demographic details were obtained.

Informed consent: The purpose of the study was explained to the subjects after which a signed consent was obtained.

Sample size and assessment: 200 patients were included as a final sample size, attending the dental institute. Post hoc comparison test was used to examine the responses for the questionnaire. A 'p' value 0.05 was considered statistically significant.

Questionnaire: A self-administered questionnaire was provided to the patients in the vernacular language and included 20 questions. The questions 1 to 5 were based on the misconceptions regarding dental caries. The questions 6 to 10 were about the myths regarding dental treatments. The questions 11 and 15 were related to the myths regarding oral cancer. Questions 16 and 20 were based on the miscellaneous misconceptions. (Fig. 1)

\section{QUESTIONNAIRE REGARDING DENTAL HEALTH CARE}

NAME:

GENDER: M / F

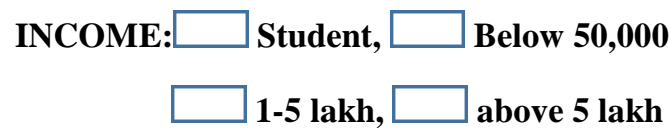

AGE:

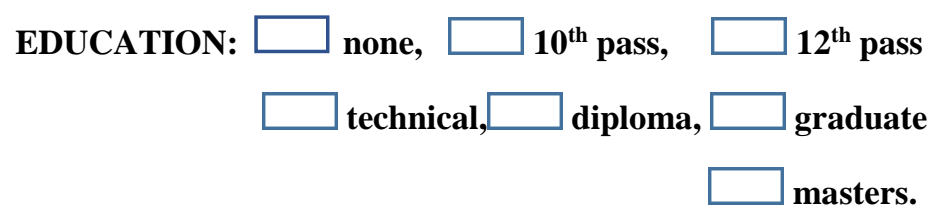

\section{QUESTIONNAIRE}

\section{QUESTIONS REGARDING DENTAL PROBLEMS:}

a. The only cause of cavities is eating sweets:
i. Agree
ii. Disagree

b. If you keep a clove besides a painful tooth, the pain is relieved:
i. Agree
ii. Disagree

c. If milk teeth are decayed, there is no need to save it as its going to fall anyways:
i. Agree
ii. Disagree

d. Extraction of wisdom teeth leads to loss of wisdom:
i. Agree
ii. Disagree

e. Flossing of teeth harms the gums:
i. Agree
ii. Disagree

II. QUESTIONS REGARDING ORAL CANCER:

a. Tobacco chewing helps in removing bad odor from the mouth:
i. Agree
ii. Disagree

b. Occasional tobacco chewing or smoking cannot harm you:
i. Agree
ii. Disagree

c. Smoking bidi helps in digestion:
i. Agree
ii. Disagree

d. Toothache is relieved by chewing tobacco:
i. Agree
ii. Disagree

e. Tobacco helps in preventing tooth decay:
i. Agree
ii. Disagree

\section{QUESTIONS REGARDING DENTAL TREATMENTS:}

a. There is no need to visit a dentist, if you have no toothache:
i. Agree
ii. Disagree

b. If you undergo scaling, your teeth will become mobile and gaps will be created between the teeth:
i. Agree
ii. Disagree

c. If you go for extraction of any of your upper teeth, it will effect your vision:
i. Agree
ii. Disagree 
d. If your tooth is treated once, there is no recurrence of tooth decay in that particular tooth:
i. Agree
ii. Disagree

e. All dental treatments are painful:
i. Agree
ii. Disagree

\section{MISCELLANEOUS QUESTIONS :}

a. Spacing in upper front teeth is considered lucky:
i. Agree
ii. Disagree

b. One should not brush teeth after delivery:
i. Agree
ii. Disagree

c. Brushing teeth for a long time and with a hard bristle brush makes teeth more white:
i. Agree
ii. Disagree

d. No dental treatment should be done during pregnancy:
i. Agree
ii Disagree

e. Cleaning teeth with salt, lemon and charcoal makes teeth white and shiny:
i. Agree
ii. Disagree

Fig. 1: Questionnaire

\section{Results}

Out of 200 out-patients who participated in the study, 98 were males and 102 were females. The participants were divided into different age groups in which Group I $=0-29$ years, Group II $=30-40$ years, Group III $=41-50$ years and Group IV $=>50$ years. The education category consisted of 3 groups in which Group $\mathrm{I}=$ illiterate, Group II = 12th pass, Group III = higher education. The income group comprised of 3 groups which were subdivided as Group I= with no income, Group II= income below 50,000 per annum and Group
III= income above 50,000 per annum.

\section{Observations in relation to gender}

$38.14 \%$ males and $40.18 \%$ females had misconceptions regarding dental problems. Out of the total respondents who had the misconceptions about oral cancer, $36.73 \%$ were males and $30.59 \%$ were females. Participants having misconceptions regarding dental treatments included $39.59 \%$ males and $47.45 \%$ females. And those who had miscellaneous misconceptions on general dental aspects included 35.92\% males and $41.37 \%$ females. (Graph 1)

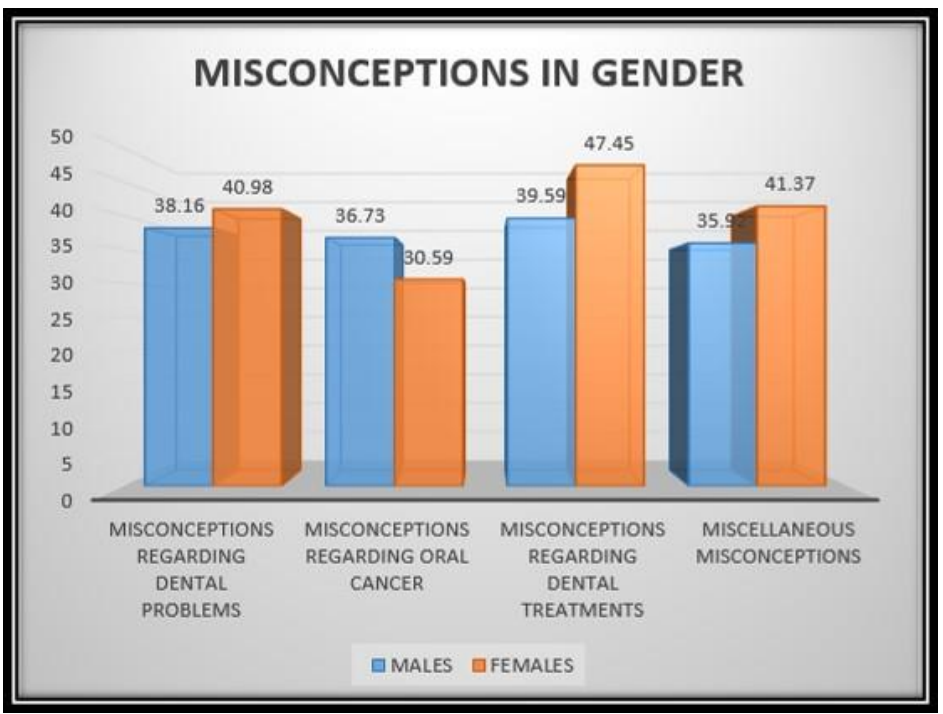

\section{Graph 1: Misconceptions based on gender}

\section{Observations in relation to age}

$37.11 \%$ participants had the misconceptions regarding dental problems in age Group I; followed by $35.90 \%$, $32.63 \%$ and $52.34 \%$ in age Groups II, III and IV respectively.

Age Group I also revealed $29.74 \%$ respondents with misconceptions regarding oral cancer followed by $24.10 \%$, $30.53 \%$ and $50.21 \%$ in age Groups II, III and IV respectively. 
$38.68 \%$ participants had the misconceptions regarding dental treatments in age Group I, $44.72 \%$ in Group II, 40.18\% in Group III and 54.04\% in Group 4.

$34.21 \%$ patients had the miscellaneous misconceptions in age Group 1, followed by $37.44 \%$ in Group II, $36.84 \%$ in Group III and $48.51 \%$ in Group IV respectively. (Graph 2)

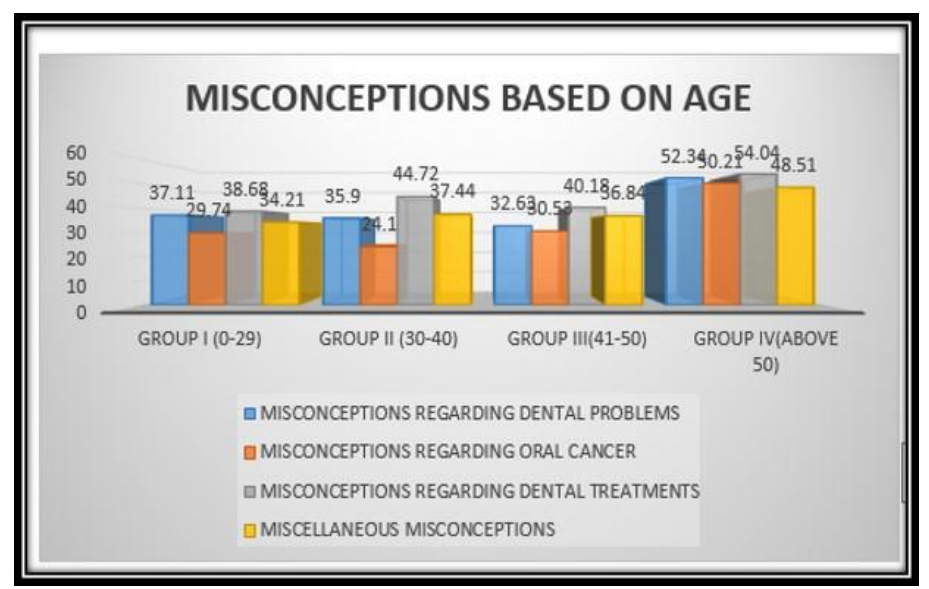

Graph 2: Misconceptions based on age

\section{Observed in relation to income}

The misconceptions regarding dental problems in Group I were $45.40 \%, 48.10 \%$ in Group II and $23.25 \%$ in Group III. Misconceptions regarding oral cancer in Group I were 34.80\%, $41.90 \%$ in Group II and $25.52 \%$ in Group III.

48\% respondents had the misconceptions regarding dental treatments in Group I, 53.33\% in Group II and 28.97\% in Group III.

43.60\% participants had the miscellaneous misconceptions in Group I, $49.05 \%$ in Group II and $23.45 \%$ in Group III. (Graph 3)

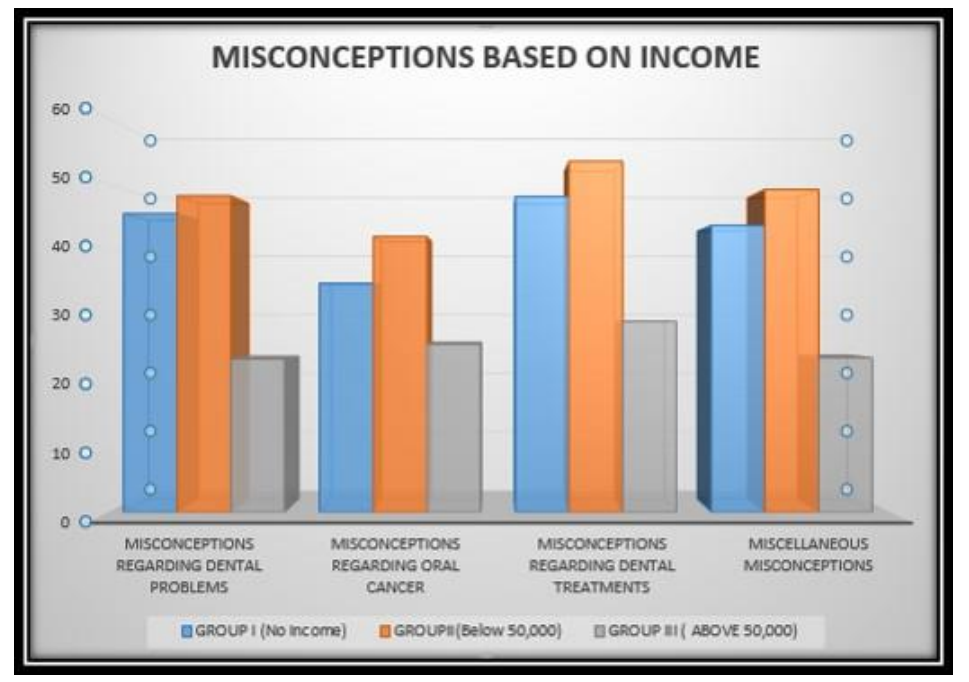

Graph 3: Misconceptions based on income

\section{Observations in relation to education}

Misconceptions regarding dental problems in Group I were 53.20\%, followed by $43.42 \%$ in Group II and 26.49\% in Group III respectively. $48 \%$ respondents had the misconceptions regarding oral cancer in Group I, 34.74\% in Group II and $22.70 \%$ in Group III.

$57.20 \%$ participants had the misconceptions regarding dental treatments in Group I, $49.21 \%$ in Group II and 49.21\% in Group III. $52.40 \%$ respondents had miscellaneous misconceptions in Group I, followed by $42.37 \%$ in Group II and $25.68 \%$ in Group III respectively. (Graph 4) 


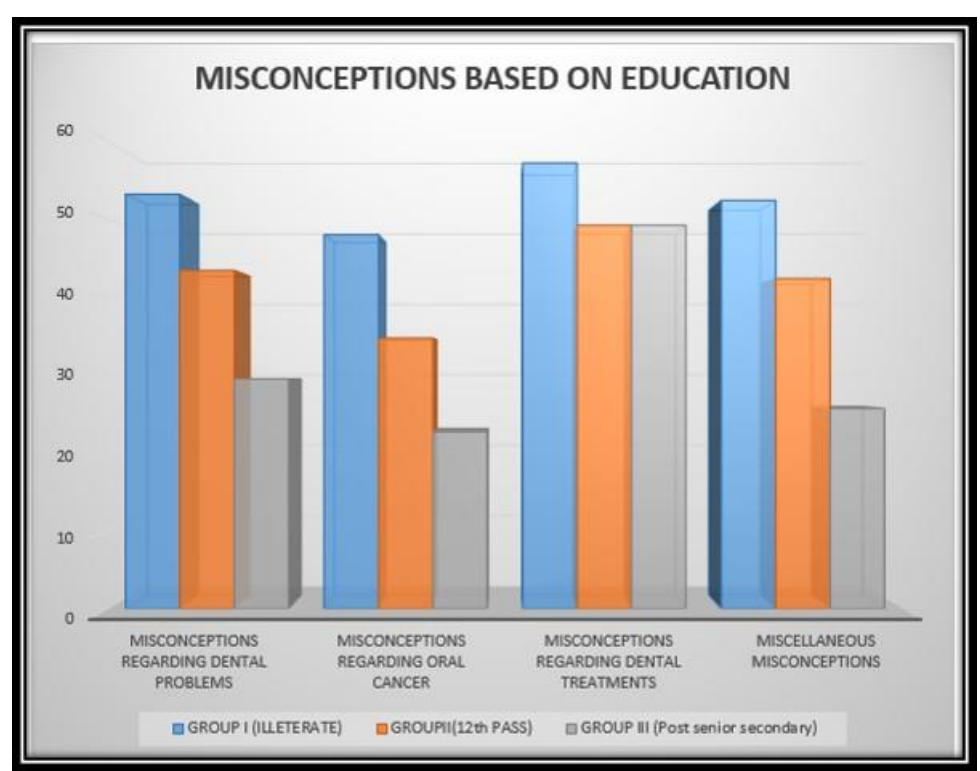

\section{Graph 4: Misconceptions based on education}

\section{Discussion}

India, a developing country is facing many challenges in rendering oral health needs. There is a very strong influence of the various misconceptions on health and dental seeking behavior in Indian population as it consists of people from different cultural backgrounds. Misconceptions play a part and parcel of everyone's life $^{3}$. Due to development and awareness systems there are more expectations of health-care facilities. We therefore need to understand the misconceptions about oral diseases in order to provide excellent care and dental education to both patients and healthy individuals. ${ }^{4}$

The present study revealed that misconceptions associated with dental treatments were significantly higher among females as compared to males. This is possibly related to the role females continue to play as the bearers of family traditions. The results of our study were similar to the study done by Thomas et al which revealed $90 \%$ of females had more misconceptions regarding dental treatments than males. ${ }^{2}$

Participants in age group above 50 years had more misconceptions than other age groups in our study. The results were same as the study done by Thomas et al which revealed $87.3 \%$ people above 50 years of age had more misconceptions. Age was also associated with ascribing importance to misconceptions, with older adults more likely to hold erroneous views on effective preventive methods. This is perhaps more understandable, but again of concern when considered in conjunction with the increasing risk of dental disease in the increasingly dentate older adult population. ${ }^{2}$

Also, in our study, misconceptions regarding dental problems, oral cancer, dental treatments and miscellaneous needs were seen more in illiterate and less educated people. Lower educational attainment was associated with a higher frequency of crediting misconceptions. This was consistent with other studies which have shown associations between lower educational attainment and poorer health outcomes, and lower educational attainment and lower utilization of health services. The finding in this study are consistent with other studies in relation to educational level and health which shows that with higher education the awareness regarding dental treatments become high. ${ }^{2}$

In the present study the misconceptions regarding dental problems, oral cancer, treatments and miscellaneous misconceptions were more evident in people earning less than 50,000 which shows that less income people like labours or workers were less aware and more prone to misconceptions. ${ }^{4,6}$

The main responsibility is on the dental community and the administrative machinery to make great efforts to achieve the following: (1) Dental awareness programs which target the rural and aged population; (2) Setting up supporting dental care facilities and dental hospitals and colleges close to rural populations; (3) Mobile dental clinics and dental camps and free dental checkups in rural setups. ${ }^{5,7}$

\section{Conclusion}

It may be concluded that majority of the respondents had various misconceptions regarding dentistry and oral cancer-related habits. Hence, it is the prime role of dentists to counsel general public in the rural and urban areas regarding misconceptions and instill awareness to resolve them.

\section{References}

1. Ain TS, Gowhar O, Sultan S. Prevalence of Perceived Myths Regarding Oral Health and Oral Cancer-causing Habits in Kashmir, India. Int J Sci Stud 2016;4(3):45-9.

2. Roberts-Thomson KF, Spencer AJ. Public knowledge of the prevention of dental decay and gum diseases. Aus Dent J 1999;44:(4):253-8. 
3. Sharma R, Mallaiah P, Marghabandhu S, Umashankar GK, Verma S. Dental myth, fallacies and misconceptions and its association with socio-dental impact locus of control scale. Int J Prevent Public Health Sci 2015;1(2):14-20.

4. Raina SA, Jain SP, Warhadpande MM. Myths and taboos in dentistry. Int J Res Med Sci 2017;5(5):1936-2.

5. Singh SV, Akbar Z, Tripathi A, Chandra S, Tripathi A. Dental myths, oral hygiene methods and nicotine habits in an ageing rural population: An Indian study. Indian $\mathbf{J}$ Dent Res 2013;24(2):242-4.

6. Tewari D, Nagesh L, Kumar M. Myths related to dentistry in the rural population of Bareilly district: A cross-sectional survey. J Dent Sci Oral Rehab 2014;5:5864.

7. Kumar S, Mythri H, Kashinath KR. Clinical perspective of myths about oral health; a hospital based survey. Univ J Pharm 2014;3:35-7. 